\title{
A Contrastive Study of Zhong Metaphors in Chinese and Middle Metaphors in English
}

\author{
Shuangshuang Lu \\ City College, Zhejiang University, China \\ Email: luss@zucc.edu.cn \\ Shenglan Tan \\ National Defense University, PLA, China \\ Email: gracetan85@hotmail.com
}

\begin{abstract}
The present study analyzes the metaphorical extensions of the Chinese spatial term zhong and the English spatial term middle. It finds that The essay finds both ZHONG and MIDDLE are found to be used in the target domains of STATES, RANK and TIME, which further provides a piece of evidence that our abstract reasoning is partly structured by our spatial thinking through schematic mapping and metaphorical extension. The present study also concludes that the spatial metaphorical system among different languages may share some cognitive universals as well as cross-linguistic variations.
\end{abstract}

Index Terms — zhong, middle, metaphor, schematic, cognitive

\section{INTRODUCTION}

The cognitive approach is drawing much attention from linguists and researchers in recent years due to its inquiry into human cognition to describe and explain linguistic phenomena. One fundamental claim of cognitive linguistics is that human beings have no access to the reality of the world independent of the conceptual structures in human minds, which are derived from and realized in bodily experience in the real world (Langacker 1987, Lakoff 1987). Thus much of the focus of cognitive linguistics is in fact on conceptual frameworks, in which metaphor and image schemas are two of the most fundamental conceptual structures. Within the studies on metaphor, special attention is given to spatial metaphors due to the primary role of spatial thinking in human cognition - "Spatial thinking is crucial to almost every aspect of our lives" (Levinson, 2003, p.1); non-spatial concepts appear to be structured by spatial conceptions in the conceptual system through special metaphors (Levinson, 2003).

In view of such a primary position of spatial thinking in human cognition, a large number of studies have been done on spatial metaphors both in English and Chinese; for instance, a study concerned with metaphors associated with up/down in English and shang (up) /xia (down) in Chinese was conducted by Lan (2002); other researches have been found focusing on the metaphors associated with up/down in English and shang/xia in Chinese (Li, 2002; Tao, 2000), front/back in English and qian (front) /hou (back) in Chinese (Wang and Han, 2008; Zhang and Luo, 2007), etc. all of which contributed to providing cross-linguistic and cross-cultural evidence for the primacy of spatial metaphors in structuring non-spatial conceptions and revealed some similarities and differences in the metaphorical extension processes cross-linguistically. However, these studies still are not conclusive and exhaustive in describing and explaining the rise of abstract concepts through metaphorical extensions from the source domain to the target domain; and more cross-linguistic and cross-cultural studies need to be conducted to conclude the existence of a universal spatial metaphorical system. One of the spatial conceptions that has been rarely explored is MIDDLE in English and ZHONG in Chinese, which is the primary concern of the present article. The present paper follows Lan (2002) in making a distinction between two linguistic terms and two conceptual structures, that is the former are referred to as zhong and middle, and the latter by $Z H O N G$ and MIDDLE.

The present article develops along the same line of the previous studies and makes its own contributions in two aspects: firstly, it analyzes the metaphorical extensions of the Chinese spatial term zhong and English spatial term middle respectively, which are important spatial concepts and lack of full explorations; thus the present study is to fill this gap and to further explore how non-spatial concepts are derived from spatial metaphorical extensions; secondly, it compares and contrasts the metaphorical extensions of two spatial terms cross-linguistically, which further provides evidence for the existence of spatial cognitive universals as well as cross-linguistic variations.

\section{CONCEPTUAL StRUCTURES, METAPHOR AND IMAGE SCHEMAS}

The present paper adopts the view of experiential realism held by cognitive linguists, which attempts to characterize meaning in terms of our physical and social experiences as human beings living in the real world. (Fauconnier, 1994; Johnson, 1987) The cognitive approach advocates that we live in the world not just as beings; we also perform and act 
in the world, perceiving and shaping the environment that surrounds us, as a result of which, we form basic conceptual structures and apply these conceptual structures to a range of more abstract domains to help us understand the abstract concepts.

A number of conceptual structures have been identified in the literature of cognitive linguistics, among which metaphor, image schemas, mental spaces etc. all receive much attention. The present paper is mainly concerned with metaphor and image schemas.

Metaphor, according to Lakoff and Johnson, plays an essential role in human conceptual categorization (1980). Metaphor is viewed in cognitive linguistics as existing extensively in ordinary language and occupies a central position in our language and thought. Metaphor enables human beings to understand one more abstract domain in terms of another less abstract domain, the former of which is referred to as the target domain and the latter the source domain (Lakoff and Turner, 1989).

Another important conceptual structure in cognitive linguistics is image schema, which has close relations with metaphor. According to Johnson (1987), an image schema is a dynamic pattern to display our perceptual interactions and thus shaped conceptual structures owing to their embodiment in our real life. It bridges a gap between bodily experience and human cognition and hence illuminates our understanding of metaphor used to help conceptualize the world (cited in Saeed, 2003). Thus image schema is viewed as a more basic level in the conceptual structure, which underlies metaphor. One way of applying image schemas into more abstract domains so as to enable human beings to understand the abstract domains is by metaphorical extension.

\section{IMAGE SCHEMAS OF ZHONG IN CHINESE AND MIDDLE IN ENGLISH}

ZHONG/MIDDLE each has their image-schematic concepts and can be delineated by either a movement or a particular area of the trajector in relation to a landmark. When ZHONG/MIDDLE are displayed as a movement along the horizontal axis, they are referred to as dynamic ZHONG/MIDDLE (see diagram1; the dotted line referred to unfixed end-point). When they are displayed as a particular position of the trajector, they are referred to as static ZHONG/MIDDLE (see diagrams 2, 3, and 4; dotted line referred to unfixed area)

The dynamic ZHONG/MIDDLE are shown in the following examples. From these examples, one can see that ZHONG/MIDDLE tend to underline an ongoing process.

(1). Feiji zai feixing zhong.

plane at flying middle

The plane is flying.

(2). Lvyou tuzhong, youren xiang tamen zuoguo jieshao.

travelling on the way, somebody to they did introduction.

Somebody told them when they were traveling.

(3). Tamen zai lunshuzhong wang'le jiben de qianti.

they in arguing forgot basic of premises.

They forgot the basic premises while arguing.

(4). The other hangs in midair as if he had been caught in the middle of running.

(5). They are in the middle of passing bills restricting predatory lending.

(6). We're in the middle of an interview.
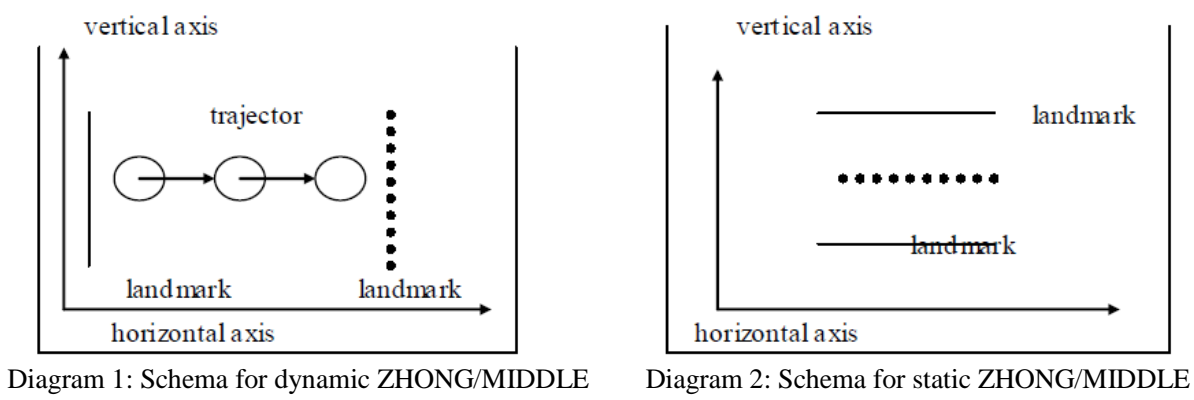


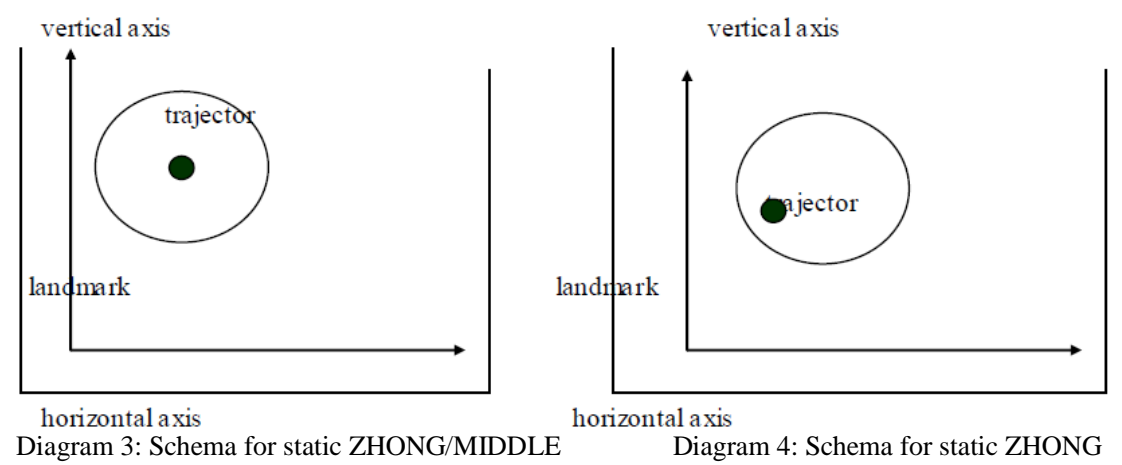

When ZHONG/MIDDLE are static, they have the two common prototypical models, the first of which describes a concept of "a position or part which is at an equal distance from two points", called "in-between" ZHONG (see diagram 2) and the second refers to "a position or part which is at an equal distance from more than two points", called "center ZHONG" (see diagram 3). The examples of this static type are given below:

(7). Taxiang ba zhetiao guanggaofangzai baozhi zhongfeng.

he wants prep. this piece advertisement newspaper center-seam

He wants to put this advertisement at the center-seam of the newspaper.

(8). Zhongqiujie jiuyao daole.

mid-autumn festival is about to arrived

Mid-autumn festival is approaching.

(9). Tade xingzhi bianpineryuan, zhongjian you kong.

Its shape and material flat and round middle have holes.

It is flat and round with holes in the middle.

(10). Zhongzhong meide shi ta chengle shejiao de zhongxin.

Various moral excellence make him became social intercourse of center

Many pleasant virtues make him the focus of the attention in the social life.

(11). In the middle of the Hall behind her a Couple exits a Room.

(12). In the middle of the night, two boys woke up and began shouting.

(13). The cake is frozen in the middle.

In addition, the fourth model is characteristic of ZHONG. It describes a concept of "within a container" belonging to containment schema (see diagram 4), which MIDDLE lacks.

(14). Tajiao wo fang zai shizhong de shafa pangbian.

he asked me put at in the room of sofa side

He asked me to put it by the sofa in the room.

(15). Xinzhong mei you qiangua.

In the heart no have worry.

There is nothing to worry about.

Whether ZHONG/MIDDLE are referred to as dynamic or static, their meanings in the above instances all show much extension from their prototypical spatial concepts to the characterization of other domains (see examples 2, 3, 5, 6, $8,10,12$ and 15 , no extension of the prototypical model shown in diagram 4 has been found in the data.). Accordingly, the following analysis will be in answer to the three questions: first, what are the extensions developed out of the prototypical models; second, how are the extensions of these two concepts evolved; and third, what are the similarities and differences between the ways in which the metaphorical use of these two concepts is developed.

\section{Methodology}

The data of the current study is retrieved from the two well-recognized corpora. One is the Chinese corpus called Peking University Modern Chinese Corpus composed of nearly 265 million Chinese characters, including two sub-corpora, namely Modern Chinese and Contemporary Chinese, both of which cover various fields such as literature and practical writing with respect to the style of the text. The corpus spanning scores of years (from 1919 to 2002) puts its focus on the language data in the recent 20 years, which is updated every year (most recent till 2005). The other is Corpus of American English containing more than 360 million words including the fields of spoken, fiction, magazine, newspaper and academic, all of which date from 1997 to 2007. Both corpora are free of charge and accessible on line.

From the Chinese corpus, the frequency of zhong is found to be $1,354,405$, taking up $0.5 \%$ of the total bank of characters. The English middle appears 65,441 times, accounting for $0.018 \%$ of the entire English corpus. For the convenience of both quantitative and qualitative analysis of the data in the present paper within the limited space, 26,055 zhong were retrieved from the Chinese corpus by focusing on the sub-corpus Modern Chinese, also most up-to-date, whereas 50,271 middle were retrieved from the English corpus corresponding to the way Chinese zhong is retrieved. 
About $0.5 \%$ of middle was selected with a preference for recency from the total data that had been retrieved. In other words, the selected data were largely recorded in 2007. From the total data of zhong that had been retrieved, around $1 \%$ of them was selected carefully with concern about the inclusion of the major fields that the sub-corpus covers. All those proper nouns that are tinted with cultural characteristics were excluded. For example, the Chinese name for China and all others associated with the name like Zhongguo (China), zhonghua (original name for China), zhongyi (traditional Chinese medicine), zhongzhuang (traditional Chinese clothes), zhonggong (Chinese Communist Party), and the person's name Sun zhongshan. In the similar vein, proper nouns associated with middle such as the Middle East are not regarded as part of the data either for the present study. Finally, the present study focuses on a database that consists of 205 zhong in Chinese and 210 middle in English.

All the instances of zhong/middle included in the database of the present paper are analyzed in terms of prototypical model of the source domains, their extensions to the target domains and how the two are mapped onto each other. In addition, generalizations are made with regard to the major target domains into which the database can be grouped.

\section{RESULTS AND DisCUSSION}

\section{A. Quantitative Analysis of $Z H O N G$}

ZHONG has its origin as spatial concepts just like SHANG and XIA (Lan, 1999, 2002). It was traditional in ancient times to insert the flag of a society in the center of a land to assemble the people when important events took place. The pictographic character symbolizes the figure and the rotation of the flag (Zhang, 2007). The flag had a center when rotating and certainly was inside the circle that it rotated. Therefore, ZHONG has originally been depicted as "center' and "inside", the evidence of which can be found on the inscriptions on oracle bones in Shang Dynasty and on ancient bronze objects in Zhou Dynasty.

Evidence from the present data finds that only $4.9 \%$ of 205 instances of zhong are used dynamic. This shows that whether in terms of its prototypical or metaphorical meanings, zhong is predominantly used to depict a static concept. Within the static $Z H O N G$, the inside sense takes up $75.1 \%$. This shows that, prototypically or metaphorically, $Z H O N G$ is primarily used to describe containment concept, which might be remarkably different from MIDDLE since the latter doesn't have the prototype of inside sense. The detailed percentages of the prototypical models are shown in Table 1.

A further examination of the Chinese database demonstrates that ZHONG can be mainly used in the following target domains: RANGE, STATE, RANK and TIME. The specific metaphorical extensions and the percentages are presented in Table2.

TABLE 1:

THE PROTOTYPICAL MODELS OF ZHONG

\begin{tabular}{|c|c|c|c|c|}
\hline Prototype model & Sub-category & Number & percentage & \\
\hline Dynamic $Z H O N G$ & & 10 & 10 & $4.9 \%$ \\
\hline \multirow[t]{3}{*}{ Static $Z H O N G$} & inside $Z H O N G$ & 154 & $75.1 \%$ & \\
\hline & center $\mathrm{ZHONG}$ & 9 & $4.4 \%$ & \\
\hline & in-between $\mathrm{ZHONG}$ & 32 & $15.6 \%$ & \\
\hline Total & & 205 & $100 \%$ & \\
\hline
\end{tabular}

TABLE 2:

\begin{tabular}{lllll}
\multicolumn{5}{c}{ THE METAPHORICAL EXTENSIONS OF ZHONG } \\
\hline $\begin{array}{l}\text { Target } \\
\text { domain }\end{array}$ & Metaphorical & Number & $\begin{array}{l}\text { Percentage } \\
\text { of } 126\end{array}$ & Percentage of 205 \\
RANGE & In the range & 56 & $44.4 \%$ & $27.3 \%$ \\
STATES & $\begin{array}{l}\text { Being in a process between the beginning and } \\
\text { ending of an event; or being in a static state. }\end{array}$ & 36 & $28.6 \%$ & $17.6 \%$ \\
& An intermediate position in a ranked scale & 25 & $19.8 \%$ & $12.2 \%$ \\
RANK & Between two temporal points & 9 & $7.2 \%$ & $4.4 \%$ \\
TIME & & 126 & $100 \%$ & $61.5 \%$ \\
\hline
\end{tabular}

As can be seen from Table2, about $61.5 \%$ (126) of the 205 occurrences are used metaphorically, slightly more than those of non-metaphorical use. This tells us that ZHONG carries its prototypical meanings by a considerable amount in comparison with other spatial concepts like SHANG/XIA (Lan, 2003). Among all the target domains, the dominant target domain is RANGE, which is extended from the prototypical concept of "inside".

\section{B. Qualitative Analysis of $\mathrm{ZHONG}$}

The above analysis answered the research question of what the metaphorical extensions of ZHONG are and what domains those extensions are used. The second question, how and on what experiential foundation those extensions are realized in the target domains, leads to this section. The four domains will be covered in turn in the following discussion.

1) RANG

ZHONG in this domain indicates the meaning of within a range.

ZHONG literally means inside, which accords with containment schema. This is reflected in human body. Human beings regard themselves as a container, both inhaling and exhaling out of their primitive living need. The concept is 
then extended to other abstract concepts and concrete beings. The abstract concepts are like human's knowledge, perception and reasoning about their inner world and outside world. The concrete beings include all those inscribed with human influence. The examples of this type are given below:

(15) Haizi de shijie zhong meiyou yisi lequ.

child of world in have no a little joy

There is no joy in this child's life.

(16) Wo zhong zai jieshi xinzhong yituan.

I important in explain in the heart doubts and suspicions

What I am concerned is how to explain my doubts and suspicions.

(17) Yexu Tianjian zhi dang ta shi nvpengyou zhong de yige.

Perhaps Tianjian only as her is girlfriend in of one

Perhaps Tianjian treats her as only one of his girlfriends.

2) STATES

ZHONG in States can express the meaning of being in a static state or being in a process of a continuous state.

ZHONG has the literal sense of in-between, i.e. between the two extremes. Just as spatial concepts SHANG and XIA are gained out of our bodily experience (Lan, 2003), ZHONG is of the same case. It is between the head and the feet, roughly in the middle of the body. When this concept is extended to everyday life, we regard a certain action as a process having a beginning and an end. Zhong is then used to express in the process of doing something, viz. in a continuous state.

We extend the containment schema of ZHONG to the descriptions of the quality or our feelings and opinions of a certain thing, treating the latter as a container. When in this container, we refer to in a static state, meaning with or influenced by this container.

(18) Waimian de anzhong wuran qile jixiang de jiaohan.

outside of in secret suddenly rose loud of gun

There is a sound of gun suddenly coming from the outside.

(19) shenghuo zaigujizhong, mei you xiao banlv.

live in loneliness have no young companion

live lonely without companionship

3) RANK

Zhong is used to describe a balance between or in the middle of two extremes such as neither

large nor small, neither good nor bad, neither high nor low.

Human beings need to categorize as well as measure things of the world in front of them. The spatial concepts SHANG, ZHONG and XIA out of bodily experience can be used to express highness, middle, and lowness of the rank. This is extended in describing different ranks of social position, educational level, political bureau, etc.

(20) dang wo chu jin zhongxue dushushi

when I early enter middle school study time

when I first went to the middle school

(21) zhong xia deng shehui

middle low class society

the middle-lower society

(22) gonggu lianhe zhongnong

consolidate unite middle peasants

consolidate and unite those middle peasants

4) TIME

Zhong is used to express somewhere between two temporal points.

This usage is still originated from the sense of in-between. In order to locating themselves temporally, human beings thought up different criteria to measure time. All these criteria provide ranks of temporal points and such temporal concepts as the beginning, the middle and the end are formed. In this way, human beings are better equipped with concrete concepts to understand each other, for instance, when they refer to things that are removed from the immediate situations.

(23) zhongwu yu zhi

noon rain stop

The rain stopped at non

(24) chile wufan shui zhong jiao

ate lunch sleep middle sleep

take a nap after lunch

\section{Quantitative Analysis of MIDDLE}

Similar to ZHONG in Chinese, the schematic models of MIDDLE describe either the process or the location of a trajector, the former of which is referred to as the dynamic MIDDLE and the latter static MIDDLE. Among the 210 occurrences of middle analyzed, $9 \%$ are found to be dynamic, leaving the rest to be static. This indicates that $M I D D L E$ 
is much more often used to depict a location rather than a motion of a trajector either in the source domain or in the target domain. Within the static models, $61.4 \%$ of them denote the prototypical spatial meaning of being in a point that is equal distant from two ends Another $28.6 \%$ of middle indicate the prototypical spatial meaning of being in the center of an entity. The detailed percentages of the dynamic and static models of MIDDLE are presented in Table 3.

Evidence from the English corpus indicates that these basic prototypical models are mainly extended into three target domains, namely, the domain of time, rank, and states. The specific percentages of the metaphorical extensions are presented in Table4.

TABLE3:

THE PROTOTYPE MODELS OF MIDDLE

\begin{tabular}{|c|c|c|c|c|}
\hline $\begin{array}{l}\text { Prototype } \\
\text { model }\end{array}$ & & Number & Percentage of 210 & \\
\hline Dynamic & & 19 & $9 \%$ & \\
\hline \multicolumn{5}{|l|}{ MIDDLE } \\
\hline Static & Center MIDDLE & 60 & $28.6 \%$ & \\
\hline$M I D D L E$ & In- between $M I D D L E$ & 150 & $61.4 \%$ & \\
\hline Total & & 210 & $100 \%$ & \\
\hline \multicolumn{5}{|c|}{$\begin{array}{c}\text { TABLE4: } \\
\text { THE METAPHORICAL EXTENSIONS OF MIDDLE }\end{array}$} \\
\hline Target domain & Metaphorical extension & Number & Percentage of 120 & $\begin{array}{l}\text { Percentage of } \\
210\end{array}$ \\
\hline STATES & $\begin{array}{l}\text { Being in a process between the beginning and } \\
\text { ending of an event; or being in a static state. }\end{array}$ & 25 & $20.8 \%$ & $11.9 \%$ \\
\hline RANK & An intermediate position in a ranked scale & 60 & $50 \%$ & $28.6 \%$ \\
\hline TIME & Between the beginning and ending of a time period & 35 & $29.2 \%$ & $16.7 \%$ \\
\hline Total & & 120 & $100 \%$ & $56.3 \%$ \\
\hline
\end{tabular}

As can be seen from Table5, 56.3\% of middle that have been analyzed carry the metaphorical meanings, which is almost balanced with $61.5 \%$ of metaphorical use of zhong in Chinese.

\section{Qualitative Analysis of MIDDLE}

The following section discusses the detailed metaphorical extensions of MIDLE in each target domain and describes the realizations of the metaphorical extensions in real life.

1). STATES:

MIDDLE denotes the meaning of being in a process between the beginning and the ending if an event; or being in a static state. Below are some examples from the data analyzed:

(25) A truck is in the middle of off-loading its supply of tainted blood.

(26) Robert Lafontaine is right in the middle of getting changed in the background.

(27) I'm in the middle of a lecture.

(28) We're in the middle of the biggest shift in 200 years

2) RANK:

MIDDLE indicates an intermediate position in a ranked scale. Its realizations found in the data can be grouped into social status, quality, quantity, sequence, education level, age etc. To be more specific, middle is between a higher and a lower social status, between better and worse qualities, between larger and smaller quantities, between the first and the last in a sequence, between elementary and advanced education levels, between youth and old etc.. Below are some examples:

(29) People in the middle class have suffered in silence too long.

(30) It is acknowledged that there are broad upper, middle, and lower levels in the market,

(31) These challenges are available for elementary, middle, and high school.

(32) Santa Barbara has been somewhat ignored, much like the middle child in many families.

(33) He couldn't find his good fastball until the middle innings.

(34) Some studies suggest that dealers are likely to be middle age.

3) TIME:

MIDDLE denotes a specific time period or specific point that is preceded and followed by a same amount of time. Unlike ZHONG in Chinese, Middle in English does not denote the meaning of within a certain time period. Below are some specific examples:

(35) It's the middle of the school year.

(36) It's the middle of February

\section{CONCLUSION}

From the above analysis based on Chinese and English data, we can see that ZHONG and MIDDLE share a great number of similarities in their metaphorical extensions. Firstly, both ZHONG and MIDDLE are found to be used in the target domains of STATES, RANK and TIME. Secondly, in the three target domains, both ZHONG and MIDDLE 
denote the meaning of "in between"; in other words, the metaphorical extensions of both ZHONG and MIDDLE are oriented by the same image schematic concept of "between two extremes" rather than "at the center of an entity". Thirdly, the percentages of metaphorical extensions of ZHONG and MIDDLE are found to be comparable, with the metaphorical use of ZHONG taking up $61.5 \%$ and that of MIDDLE accounting for 56.3\%. Fourthly, both ZHONG and MIDDLE are found to be predominantly used in their static models, which describe the position or location of a trajector.

The analysis also reveals some differences between ZHONG and MIDDLE both in their prototypical models and metaphorical extensions. Firstly, the prototypical models of ZHONG cover the sense of "inside", which is not covered by the schematic concepts of MIIDLE. Secondly, ZHONG is found in the target domain of RANE to denote the meaning of within a certain range while no evidence in the English data shows this use of MIDDLE. Secondly, the frequency distribution of metaphorical extensions of ZHONG and MIDLE reveal some differences: the most frequently occurring metaphorical extension in ZHONG is found in the domain of RANG, followed by STATES, RANK and TIME while in MIDDLE, the most frequently occurring metaphorical extensions are found in the domain of RANK, which is followed by TIME and STATES.

In conclusion, the present study further provides a piece of evidence that our abstract reasoning is partly structured by our spatial thinking through schematic mapping and metaphorical extension, which is evidenced by the fact that in both Chinese and English, abstract concepts such as RANK, TIME, and STATES are structured by schematic models of ZHONG and MIDDLE. The present study also concludes that the spatial metaphorical system among different languages may share some cognitive universals as well as cross-linguistic variations.

Notes:

1. According to Brugman and Lakoff, ZHONG/MIDDLE (1988, cited in Saeed, 2003)) have their central prototypical meaning, i.e. "center". The other models are related senses extended from the central prototype. As the other two models of ZHONG and three models of MIDDLE all have their extended metaphorical extensions, here the central sense and their related senses are all treated as equally prototypical.

2. It is claimed at their website to be updated every year, but for some unknown reason, the most recent is 2005 .

\section{REFERENCES}

[1] Coulson, S. (2001). Semantic Leaps. Cambridge: Cambridge University Press.

[2] Fauconnier, G. (1994). Mental Spaces: Aspects of Meaning Construction in Natural Language. Cambridge: Cambridge University Press. (republished by Beijing: World Publishing Corporation, 2008).

[3] Johnson, M. (1987). The body in the Mind. Chicago: University of Chicago Press.

[4] Lakoff, G. (1987). Women, fire, and dangerous things. Chicago: University of Chicago Press.

[5] Lakoff, G. and Johnson, M. (1980). Metaphors We Live By. Chicago: University of Chicago Press.

[6] Lakoff, G. and Turner, M. (1989). More than Cool Reason: a Field to Guide to Poetic Metaphor. Chicago: University of Chicago Press.

[7] Langacker, R. (1987). Foundations of Cognitive Grammar. Standford: Standford University Press.

[8] Lan, C. (1999). A cognitive approach to spatial metaphors in Chinese. Foreign Language Teaching and Research. 120 (4), 7-15.

[9] Lan C. (2002). A cognitive approach to Up/Down metaphors in English and Shang/Xia metaphors in Chinese. In Altenberg, B., and Granger S. (Ed). Lexis in Contrast. Corpus-based approaches. Philadelphia, PA, USA: John Benjamins Publishing Company, 161-184.

[10] Levinson, S.C. (2003). Space in Language and Cognition: Explorations in Cognitive Diversity. Cambridge: Cambridge University Press.

[11] Li, Y. (2002). On Orientational Metaphor of 'Up and Down'. Journal of Chongqing Jiaotong University. 2(3), 59-61.

[12] Saeed, . J.I. (2003). Semantics. Blackwell Publishing Ltd.

[13] Tao,W. (2000). On Spatial and Metaphorical Cognition of up. Foreign Language Research 102 (4), 13-17.

[14] Wang, L and Han, Y. (2008). A Study of Spatial-Temporal Metaphors of Qian/Hou in Chinese. Chinese Teaching and Research, 72-73.

[15] Zhang, J and Luo, R. (2007). An In-Depth Chinese-English Contrastive Study on Spatial-Temporal Metaphor. Foreign Language Research, 135(2), 68-73.

[16] Zhang, H. (2007). on Zhong. Journal of Xiamen University, 181(3), 101-106.

Shuangshuang Lu was born in Zhejiang, China. She received her BA degree in English language and literature from Beijing Normal University, China in 2007 and she received her MA degree in English linguistics from Beijing Foreign Studies University, China in 2010. She is currently an EFL teacher at Zhejiang University City College. Her research interests include discourse analysis, systemic functional linguistics and applied linguistics.

Shenglan Tan was born in Anqing, Anhui Province. She received her M.A. degree in 2010 majoring in Linguistics and Applied Linguistics from Beijing Foreign Studies University, PRC. She is currently an interpreter and translator with the College of Defence Studies, National Defense University, PLA, PRC. Her research interests are classroom discourse, political discourse and military discourse. 